\title{
Transtornos mentais comuns em residentes de enfermagem: uma análise a partir do Self Reporting Questionnaire
}

\author{
Mental disorders common among nursing residents: an analysis based on the Self-Reporting Questionnaire
}

Trastornos mentales comunes en residentes de enfermería: un análisis a partir del Self Reporting Questionnaire

\author{
Camila Biscacio Falco'; Janaina Mengal Gomes Fabril'; Elias Barbosa Oliveirall'; Alexandre Vicente Silva"v; \\ Magda Guimarães de Araújo Faria ${ }^{v}$; Célia Caldeira Fonseca Kestenberg ${ }^{V I}$
}

\begin{abstract}
RESUMO
Objetivos: verificar a suspeição de transtornos mentais comuns (TMC) em residentes de enfermagem e analisar os fatores preditores. Método: estudo transversal com uma amostra de 130 residentes de um hospital universitário, situado no município do Rio de Janeiro, que responderam ao Self Reporting Questionnaire (SRQ-20) e questões sociodemográficas, em 2018. Os dados foram analisados por meio da estatística descritiva. Estudo aprovado por Comitê de Ética em Pesquisa. Resultados: a amostra foi composta, em sua maioria, por jovens, solteiros e do sexo feminino, cuja suspeição de transtornos mentais comuns atingiu $52 \%$. Dos fatores preditores mais frequentes, $87,53 \%$ afirmaram sentirem-se nervosos, tensos e preocupados, $60,7 \%$, cansados o tempo todo e $57,7 \%$ dormem mal. Conclusão: a suspeição de TMC na amostra encontra-se bem acima de estudos realizados com profissionais da saúde e áreas afins, cujos fatores preditores evidenciam sofrimento psíquico e risco de evolução para quadros psiquiátricos severos.
\end{abstract}

Descritores: Enfermagem; saúde mental; saúde do trabalhador; especialização.

\begin{abstract}
Objective: to detect suspected common mental disorders (CMDs) in nursing residents, and identify related predictive factors. Method: this cross-sectional study examined a sample of 130 residents at a Rio de Janeiro city university hospital who answered the Self Reporting Questionnaire (SRQ-20) and sociodemographic questions, in 2018. The resulting data were analyzed using descriptive statistics. The study was approved by the research ethics committee. Results: the results revealed suspected CMDs in $52 \%$ of the sample, which was mainly young, single and female. Of the frequent predictors, $87.53 \%$ said they felt nervous, tense and worried, $60.7 \%$ felt tired all the time, and $57.7 \%$ slept poorly. Conclusion: the level of suspected CMDs in the sample was well above those found in studies of professionals of the health and related fields, and the predictors revealed psychological distress and risk of evolution to severe psychiatric conditions.

Descriptors: Nursing; mental health; worker's health; specialization.
\end{abstract}

\section{RESUMEN}

Objetivo: verificar la sospecha de trastornos mentales comunes (TMC) en residentes de enfermería y analizar los factores predictores. Método: estudio transversal con una muestra de 130 residentes de un hospital universitario, situado en el municipio de Río de Janeiro, que contestaron al Self Reporting Questionnaire (SRQ-20) y cuestiones sociodemográficas, en 2018. Los datos fueron analizados por medio de la estadística descriptiva. El estudio fue aprobado por el Comité de Ética en Investigación. Resultados: la muestra estaba compuesta, en su mayoría, por jóvenes, solteros y del sexo femenino, siendo que del $52 \%$ se sospechaba trastornos mentales comunes. En cuanto a los factores predictores más frecuentes, un $87,53 \%$ afirmó sentirse nervioso, tenso y preocupado, el 60,7\% sentirse cansado todo el tiempo y el 57,7\% dormir mal. Conclusión: la sospecha de TMC en la muestra está muy por encima de los estudios realizados junto a profesionales de la salud y áreas relacionadas cuyos predictores evidencian sufrimiento psicológico y riesgo de evolución hacia cuadros psiquiátricos severos.

Descriptores: Enfermería; salud mental; salud del trabajador; especialización.

\section{INTRODUÇÃO}

Para a Organização Mundial de Saúde (OMS) existe um número expressivo de trabalhadores com transtornos mentais comuns (TMC) na área de serviços, incluindo os profissionais da saúde, em função das mudanças nos processos laborais, diminuição dos postos de trabalho e o uso de novas tecnologias que alteraram de modo substancial os sistemas produtivos devido à exigência de rapidez, intensificação do trabalho e polivalência ${ }^{1}$. No setor saúde, a enfermagem representa a segunda maior força de trabalho, cujos profissionais, em sua maioria, atuam na atenção curativa, principalmente no setor hospitalar. Esses profissionais além de conviverem diuturnamente com o sofrimento humano, enfrentam problemas como a dupla jornada de trabalho, os baixos salários, a terceirização com perda da estabilidade empregatícia, desvalorização e condições inadequadas de trabalho; fatores que em seu conjunto acarretam prejuízos à saúde física e mental da categoria².

\footnotetext{
IEnfermeira. Especialista em Enfermagem Psiquiátrica e Saúde Mental. Universidade do Estado do Rio de Janeiro. Brasil. E-mail: mila.biscacio@hotmail.com "Enfermeira. Mestre em Enfermagem. Professora Assistente, Universidade do Estado do Rio de Janeiro. Brasil. E-mail: janamgfabri@gmail.com "'Enfermeiro. Doutor em Enfermagem. Professor Associado, Universidade do Estado do Rio de Janeiro. Brasil. E-mail: eliasbo@oi.com.br IVEnfermeiro. Doutor em Enfermagem. Professor Assistente, Universidade do Estado do Rio de Janeiro. Brasil. E-mail: alexvicentesilva35@gmail.com vEnfermeira. Doutora em Enfermagem. Professora Adjunta, Universidade do Estado do Rio de Janeiro. Brasil. E-mail: magda.faria@live.com

viEnfermeira. Doutora em Enfermagem. Professora Adjunta, Universidade do Estado do Rio de Janeiro. Brasil. E-mail: celiaprofuerj@gmail.com
} 
Pesquisa com trabalhadores de enfermagem de um hospital universitário evidenciou que esses profissionais atingem elevado índice de adoecimento e afastamentos por doenças osteomusculares, seguidas de transtornos mentais, sendo imperativo intensificar, junto a eles, medidas protetoras para a promoção da saúde mental e melhora das condições laborais ${ }^{3}$. Dentre as doenças ocupacionais, os transtornos mentais são desconhecidos e subestimados por empregadores e trabalhadores, o que caracteriza significativa dificuldade no seu efetivo enfrentamento. Há necessidade de maior visibilidade desses transtornos, no intuito de investigá-los e preveni-los adequadamente, com vistas à saúde mental do trabalhador, que vem sendo diuturnamente desestabilizado no mundo contemporâneo do trabalho ${ }^{4}$.

No que diz respeito aos residentes de enfermagem, principalmente os que atuam em ambiente hospitalar, por fazerem parte, mesmo que temporariamente do contingente de trabalhadores também sofrem os reflexos das limitações impostas pelo modelo neoliberal e a flexibilização das relações trabalhistas. Desse modo, a saúde e o desempenho dos residentes nos campos de atuação são afetados devido à ausência de preceptores e também no que diz respeito à precariedade das condições de trabalho. Trata-se de um coletivo de trabalhadores jovens, recémformados, com limitada ou nenhuma familiaridade com o processo de trabalho, cujo sofrimento psíquico pode ser intensificado em função da ausência de autonomia e baixo controle sobre o processo de trabalho ${ }^{5}$.

Com o propósito de subsidiar o estudo sobre os TMC em residentes de enfermagem, foi realizado o levantamento do estado da arte na Biblioteca Virtual de Saúde (BVS), no modo integrado com a Base de Dados de Enfermagem (BDENF), Literatura Latino-Americana e do Caribe em Ciências da Saúde (LILACS), Medical Literature Analysis and Retrieval System Online (MEDLINE) e na Cumulative Index to Nursing and Allied Health Literature (CINAHL). Selecionados artigos publicados prioritariamente no país cujo recorte temporal abrangeu o período de 2014 a 2018 . Constatou-se que apesar de haver produção do conhecimento sobre TMC na enfermagem e em outras áreas da saúde, existe incipiência de estudos com residentes de enfermagem.

A partir do exposto, elaborou-se o seguinte pressuposto: existe suspeição de TMC em residentes de enfermagem por serem em sua maioria do sexo feminino, jovens, inexperientes e não terem desenvolvido mecanismos adaptativos frente às exigências da formação. No intuito de contribuir com a discussão e reflexão sobre a importância da minimização do sofrimento psíquico nessa parcela de trabalhadores, o presente estudo teve como objetivos: verificar a suspeição de transtornos mentais comuns em residentes de enfermagem da área hospitalar e analisar os fatores preditores mais frequentes no grupo.

\section{REVISÃO DE LITERATURA}

Os TMC ou distúrbios psíquicos menores (DPM) são quadros de intenso sofrimento psíquico com importantes repercussões para a saúde do indivíduo e prejuízos em vários aspectos da vida em termos de desempenho de papéis, envolvendo trabalho, estudos e demais atividades do cotidiano. A expressão TMC foi cunhada por Goldeber e Huxley (1990), cujo conceito desenvolveu-se na década de 1970, por meio de pesquisas sobre o adoecimento mental no âmbito da atenção primária em saúde e caracteriza-se por sintomatologia não psicótica através de queixas de ansiedade, irritabilidade, somatização, diminuição da energia vital e humor depressivo ${ }^{1}$.

A partir dos anos 1970, a OMS preocupada com o aumento de indivíduos com transtornos mentais graves, principalmente em comunidades com baixo poder aquisitivo e vivendo em situação de vulnerabilidade social, iniciou um programa de rastreamento dos TMC na atenção primária, mediante o Self Report Questionnaire (SRQ-20). O rastreamento tinha como propósito a identificação de indivíduos em sofrimento psíquico, de modo a oferecer algum tipo de atenção em saúde mental a essas pessoas, visando a minimizar a evolução para quadros psiquiátricos graves e o uso indiscriminado de diazepínicos ${ }^{6}$.

Os TMC, apesar de não constituírem uma entidade clinica específica e expressa no nível coletivo, resultam de determinantes genéticos, comportamentais e ambientais que se associam a fatores como sexo, faixa etária, situação conjugal, condições de vida e trabalho. Estudos internacionais evidenciam a prevalência de TMC entre $24,6 \%$ a $45,3 \%$ na população geral, sendo que em amostras de municípios brasileiros as taxas variam de 17 a $35 \%{ }^{7}$.

Recente estudo de revisão integrativa da literatura nacional e internacional sobre os transtornos mentais em trabalhadores de enfermagem, em um recorte temporal de 10 anos, evidenciou que a produção do conhecimento no período pesquisado abrangeu temas como estresse, burnout e depressão. Apesar do aumento de publicações sobre aspectos relacionados à saúde mental do trabalhador de enfermagem a partir de 2009, dos 31 artigos selecionados e analisados, não houve referência aos $\mathrm{TMC}^{8}$.

\section{METOdologia}

Estudo transversal, de abordagem quantitativa, foi realizado em um hospital universitário, situado no município do Rio de Janeiro, com 130 residentes de enfermagem, após a assinatura do termo de Consentimento Livre e Esclarecido. Esclareceram-se os objetivos do estudo, a importância da participação do residente, garantiu-se o 
anonimato e ratificou-se que os participantes poderiam retirar o consentimento em qualquer fase da pesquisa. O curso de Residência de Enfermagem da instituição que serviu como campo de estudo é estruturado conforme a Resolução no 259/2001 do Conselho Federal de Enfermagem (COFEN), que regulamenta os programas de residência em âmbito nacional como modalidade de pós-graduação lato sensu ${ }^{9}$.

A amostra por conveniência ou demanda espontânea foi composta por todos residentes, sendo 66 do primeiro ano e 64 do segundo. Adotados como critérios de inclusão, os residentes que estivessem efetivamente matriculados e realizando as atividades práticas e teóricas previstas no programa. As normas éticas em pesquisa envolvendo seres humanos foram atendidas, cujo projeto foi aprovado pelo Comitê de Ética em Pesquisa do Hospital Universitário Pedro Ernesto (CEP/HUPE - parecer de número 1.687.061).

O estudo incluiu as variáveis dependentes: sexo; faixa etária, situação conjugal, se possuem filhos, renda familiar, padrão de sono, ocorrência de processo migratório, uso regular de medicações, diagnóstico de doença e as variáveis independentes segundo o Self Reporting Questionnaire (SRQ-20). A suspeição de TMC foi verificada através do Self Reporting Questionnaire (SRQ-20) validado para o contexto brasileiro ${ }^{10}$ em 1986, constituído de 20 itens com alternativas de respostas dicotômicas do tipo $\operatorname{sim}$ ou não. O ponto de corte utilizado na suspeição de TMC, foi de 8 respostas positivas para o sexo feminino e 6 para o masculino ${ }^{1,11}$.

Os dados foram armazenados no programa software Microsoft Excel para Windows submetidos à análise estatística, com cálculos de frequência absoluta e percentual das variáveis dependentes. Sobre os fatores preditores de TMC na amostra, trabalhou-se, também, com a frequência absoluta e relativa das respostas, destacando-se as afirmativas, para cada fator. Os resultados foram discutidos à luz de estudos sobre os TCM na enfermagem e em residentes da área da saúde e correlatos à saúde do trabalhador, pois não foi encontrado nenhum artigo sobre o tema em residentes de enfermagem.

\section{RESULTADOS E DISCUSSÃo}

As características sociodemográficas e clínicas da amostra são descritas a seguir,

\section{Características dos participantes do estudo}

De uma população de 130 residentes que atuavam no hospital universitário, na ocasião em que os dados foram coletados, responderam ao SRQ-20 todos os residentes do primeiro ano (50,7\%) e do segundo (49,3\%). São do sexo feminino (92,3\%), idade entre 20 a 25 anos $(40,7 \%)$, solteiros $86(66,1 \%)$, possuem filhos $(20,7 \%)$, renda familiar entre 3 a 6 salários mínimos, (70,7\%) dormem de 4 a 6 horas aproximadamente (56,9\%), realizaram processo migratório $(19,2 \%)$, faz uso regular de medicações (50\%), possuem diagnóstico médico (39,2\%), segundo a Tabela 1.

\section{Suspeição de TCM na amostra}

De acordo com os pontos de corte estabelecidos, para os sexos masculino e feminino, a suspeição global de TCM na amostra foi de 52,1\%, sendo $21,4 \%$ em residentes do primeiro ano (R1) e 30,7\% do segundo (R2). Apesar dos perfis sóciodemográficos dos R1 e R2 não terem diferenças estatisticamente significativas, infere-se que os R2, embora tenham maior experiência e vivência em relação ao processo formativo, estão mais expostos aos estressores ocupacionais, pelo fato de aturarem em setores especializados, cuidando de pacientes com maior nível de complexidade e com risco de complicações e morte ${ }^{12}$.

No presente estudo, verificou-se maior frequência de respostas afirmativas do SRQ-20 em residentes que atuavam em setores fechados e com alta densidade tecnológica e, dentre eles, o centro cirúrgico, nefrologia e terapia intensiva. Estes dados reforçam a necessidade de uma atenção especial à saúde dos especializandos desses campos, por serem ambientes insalubres e de riscos para a saúde devido a inúmeras demandas psicológicas e motoras ${ }^{13}$. Estudo transversal, que avaliou 178 residentes médicos e da área multiprofissional para detecção de TMC, verificou a prevalência global de $51,1 \%$, magnitude considerada elevada quando comparada à da população em geral. A prevalência de TMC nessa categoria foi $39 \%$ maior do que em residentes não médicos, o que pode ser explicado em parte, pelas particularidades das atividades inerentes à categoria médica e também pelo número de mulheres na profissão ${ }^{14}$.

A suspeição de TMC em uma amostra de estudantes dos Cursos de Graduação em Enfermagem e Psicologia foi de $35,7 \%$ e dentre as queixas mais frequentes evidenciou-se sentir-se tenso, nervoso, ter sentimentos de tristeza, sensações desagradáveis no estômago e cansaço o tempo todo. Tais dados evidenciam a necessidade de se investigar também as queixas isoladas dos TMC, por serem potencialmente incapacitantes ${ }^{15}$.

Estudos sobre TMC vêm apontando a importante da rede social na prevenção desse tipo de transtorno. A família possui papel relevante junto aos residentes na fase de transição do ambiente universitário para o de formação/trabalho, tendo em vista a sobrecarga do especializando em termos de compromissos assumidos, a carga horária extensa e demais atividades 
acadêmicas. Nesse sentido, identificou-se no estudo que $19,2 \%$ residentes se afastaram de suas famílias e migraram de outros estados e/ou município para cursarem a residência. A distribuição geográfica de movimentos migratórios da equipe de enfermagem indica a concentração desses profissionais nas capitais e áreas de maior desenvolvimento econômico. Esse processo pode gerar certo desequilíbrio, visto que altos níveis de migração podem causar aumento do desemprego, conflitos entre migrantes e nativos e redução da força de trabalho nas áreas de migração ${ }^{16}$.

TABELA 1: Características sociodemográficas e clinicas na amostra. Rio de Janeiro, Brasil, 2018.

\begin{tabular}{|c|c|c|c|c|c|c|}
\hline \multirow[t]{2}{*}{ Variáveis } & \multicolumn{2}{|c|}{ R1 } & \multicolumn{2}{|c|}{$\mathbf{R 2}$} & \multicolumn{2}{|c|}{ Total $(\mathrm{N}=130)$} \\
\hline & $\mathbf{f}$ & $\%$ & $f$ & $\%$ & $\mathbf{f}$ & $\%$ \\
\hline \multicolumn{7}{|l|}{ Sexo } \\
\hline Feminino & 62 & 47,7 & 58 & 44,6 & 120 & 92,3 \\
\hline Masculino & 4 & 3,07 & 6 & 4,6 & - & 7,7 \\
\hline \multicolumn{7}{|l|}{ Faixa etária } \\
\hline 20 a 25 anos & 23 & 17,7 & 30 & 23,07 & 53 & 40,7 \\
\hline 26 a 33 anos & 28 & 21,5 & 25 & 19,2 & 53 & 40,7 \\
\hline Acima de 33 anos & 15 & 11,5 & 9 & 6,9 & 24 & 18,6 \\
\hline \multicolumn{7}{|l|}{ Situação conjugal } \\
\hline Solteiro & 44 & 33,9 & 42 & 32,3 & 86 & 66,1 \\
\hline Casado/união estável & 20 & 15,4 & 20 & 15,4 & 40 & 30,3 \\
\hline Divorciado & 2 & 1,5 & 2 & 1,5 & 4 & 3,6 \\
\hline \multicolumn{7}{|l|}{ Possuem filhos } \\
\hline Sim & 16 & 12,3 & 11 & 8,4 & 27 & 20,7 \\
\hline Não & 50 & 38,5 & 53 & 40,7 & 103 & 79,3 \\
\hline \multicolumn{7}{|l|}{ Renda familiar } \\
\hline 3 a 6 salários mínimos & 47 & 36,1 & 45 & 34,6 & 92 & 70,7 \\
\hline 7 a 10 salários mínimos & 11 & 8,4 & 16 & 12,3 & 27 & 20,7 \\
\hline > 10 salários mínimos & 7 & 5,4 & 03 & 2,3 & 10 & 7,7 \\
\hline Não respondeu & 1 & 0,8 & - & - & 1 & 0,9 \\
\hline \multicolumn{7}{|l|}{ Padrão de sono diário } \\
\hline Menos de $4 \mathrm{~h}$ & 3 & 2,3 & 8 & 6,15 & 11 & 8,5 \\
\hline $4 h$ a $5 h 59$ & 36 & 27,7 & 38 & 29,2 & 74 & 56,9 \\
\hline $6 \mathrm{~h}$ a $8 \mathrm{~h}$ & 25 & 19,2 & 18 & 13,8 & 43 & 33,0 \\
\hline Mais de $8 \mathrm{~h}$ & 1 & 0,8 & - & - & 1 & 0,8 \\
\hline Não responderam & 1 & 0,8 & - & - & 1 & 0,8 \\
\hline \multicolumn{7}{|c|}{ Houve processo migratório? } \\
\hline Sim & 10 & 7,7 & 15 & 11,5 & 25 & 19,2 \\
\hline Não & 55 & 42,3 & 48 & 36,9 & 103 & 79,2 \\
\hline Não responderam & 1 & 0,8 & 1 & 0,8 & 2 & 1,6 \\
\hline \multicolumn{7}{|c|}{ Uso regular de medicações } \\
\hline Sim & 27 & 20,8 & 38 & 29,2 & 65 & 50 \\
\hline Não & 39 & 60,0 & 26 & 40,0 & 65 & 50 \\
\hline \multicolumn{7}{|c|}{ Possui diagnóstico médico? } \\
\hline Sim & 21 & 16,1 & 30 & 23,0 & 51 & 39,2 \\
\hline Não & 45 & 34,6 & 34 & 26,1 & 79 & 60,8 \\
\hline
\end{tabular}

\section{Análise dos fatores preditores de TCM na amostra}

A distribuição das respostas da amostra às questões do SQR-20, é apresentada na Tabela 2.

$\mathrm{Na}$ análise estatística dos fatores prevalentes de suspeição de TMC, verificaram-se as seguintes respostas afirmativas: Fator I - maior frequência de respostas para as questões sentir-se nervoso, tenso ou preocupado e sentir-se triste ultimamente. Fator II - sentir-se cansado o tempo todo, cansar-se com facilidade e ter dificuldades em tomar decisões. Fator III - dormir mal, ter dores de cabeça freqüentes, sentir desconforto estomacal. Fator IV - ter perdido interesse pelas coisas, se sentir incapaz de desempenhar um papel útil em sua vida e se sentir inútil na vida. Chamou a atenção, a afirmativa de quatro participantes ter pensado em dar fim a sua vida.

Identificou-se que a queixa mais frequente na amostra foi sente-se nervoso(a), tenso(a), preocupado(a) em $87,6 \%$ participantes (Tabela 2). Esses resultados são similares aos encontrados em uma amostra de 335 trabalhadores de 
enfermagem que atuavam em um hospital de grande porte. O humor depressivo ansioso foi a queixa mais prevalente, seguido de sintomas somáticos (dorme mal e tem dores de cabeça frequentemente), sendo o fator IV (pensamentos depressivos) o de menor prevalência. Esses dados devem ser problematizados e discutidas suas consequências no processo de trabalho diário dos profissionais, pois cefaleia e privação do sono podem interferir na atenção exigida no processo de cuidar e contribuir para a ocorrência de incidentes que comprometem a segurança do paciente e a do trabalhador ${ }^{17}$.

TABELA 2: Respostas às questões do Self Reporting Questionnaire. Rio de Janeiro, Brasil, 2018. (N=130)

\begin{tabular}{lcccc}
\multicolumn{1}{c}{ Fatores do SQR-20 } & \multicolumn{2}{c}{ Sim } & \multicolumn{2}{c}{ Não } \\
& f & \% & f & \% \\
\hline Fator I - Humor depressivo ansioso & & & & \\
Sente-se nervoso(a), tenso(a) ou preocupado(a)? & 114 & 87,6 & 16 & 12,4 \\
Assusta-se com facilidade? & 40 & 30,7 & 90 & 69,3 \\
Sente-se triste ultimamente? & 53 & 40,7 & 77 & 59,3 \\
Você chora mais do que de costume? & 34 & 26,1 & 96 & 73,9 \\
Fator II - Diminuição da energia vital & & & & \\
Você se cansa com facilidade? & 72 & 55,3 & 58 & 44,7 \\
Tem dificuldades em tomar decisões? & 63 & 48,4 & 67 & 51,6 \\
Tem dificuldades de ter satisfação em suas tarefas? & 54 & 41,5 & 76 & 58,5 \\
Tem dificuldade de pensar com clareza? & 50 & 38,5 & 80 & 61,5 \\
Seu trabalho Ihe causa sofrimento? & 34 & 26,1 & 96 & 73,9 \\
Sente-se cansado(a) o tempo todo? & 79 & 60,7 & 51 & 39,3 \\
Fator III - Sintomas somáticos & & & & \\
Você sente desconforto estomacal? & 44 & 33,8 & 86 & 68,2 \\
Tem falta de apetite? & 11 & 8,4 & 119 & 91,6 \\
Tem dores de cabeça frequentes? & 57 & 43,8 & 73 & 56,2 \\
Você dorme mal? & 75 & 57,7 & 55 & 42,3 \\
Tem má digestão? & 36 & 27,7 & 94 & 72,3 \\
Tem tremores nas mãos? & 22 & 16,9 & 108 & 83,1 \\
Fator IV - Pensamentos depressivos & & & & \\
Tem perdido interesse pelas coisas? & 41 & 31,8 & 89 & 68,2 \\
É incapaz de desempenhar um papel útil em sua vida? & 15 & 17,6 & 115 & 82,4 \\
Você se sente inútil em sua vida? & 9 & 6,9 & 121 & 93,1 \\
Tem pensado em dar fim a sua vida? & 4 & 3,1 & 126 & 96,9 \\
\hline & & & &
\end{tabular}

Estudo realizado com 59 enfermeiros da Estratégia de Saúde da Família evidenciou que a queixa mais referida na amostra foi ansiedade acompanhada de pensamento acelerado, demora em se desligar do serviço, tensão, agitação, palpitações e tremores. Tais sintomas foram associados à falta de estrutura e ao excesso de trabalho, frustração por não realizar tudo o que é solicitado e sensação de que somente o enfermeiro é responsável pela equipe ${ }^{18}$.

Acerca da diminuição da energia vital (Fator II), identificou-se maior frequência de respostas afirmativas para sentir-se cansado o tempo todo, cansar-se com facilidade e ter dificuldades em tomar decisões. Estes dados vão ao encontro de estudo com trabalhadores de enfermagem de um hospital universitário que verificou a ocorrência de TMC e associação com a menor capacidade para o trabalho, a percepção do estado de saúde como ruim / pior (geral e atual) e ao fato de estarem insatisfeitos com o sono, principalmente entre trabalhadores do sexo feminino ${ }^{19}$. Outros fatores devem ser considerados para maiores prevalências dos TMC e, dentre eles não possuir tempo para lazer, apresentar quatro ou mais problemas de saúde diagnosticados e insatisfação como sono ${ }^{20}$.

Ambientes de trabalho insalubres podem gerar distúrbios de ordem biopsicossocial e exacerbar o cansaço, a fadiga e o sofrimento psíquico. Acrescentam-se a rígida estrutura hierárquica, as longas jornadas de trabalho, o ritmo acelerado de produção devido à sobrecarga de tarefas, divisão fragmentada do trabalho, automação por ações repetitivas, escassez de recursos humanos e materiais e a complexidades das ações executadas, dentre outros ${ }^{21}$. $O$ excesso de trabalho, principalmente em profissionais que atuam em regime de turnos, implica em restrição dos horários destinados a atividades de grande importância na qualidade de vida, como alimentação, atividade física, cuidados com a saúde, lazer, repouso, sono e maior participação das atividades com a família; considerada uma importante rede de apoio social ${ }^{22}$. 
No que diz respeito ao Fator III (somatização), houve maior frequência de respostas afirmativas para dormir mal, ter dores de cabeça frequentes e sentir desconforto estomacal, cabendo destacar que o padrão de sono insatisfatório foi a terceira queixa mais frequente na amostra e referida por $75(57,7 \%)$ residentes, segundo a Tabela 2 . Esses dados vão ao encontro das características sociodemográficas relacionadas na Tabela 1, em que 56,9\% indicaram dormir aproximadamente de 4 a 6 horas por dia. Trabalhadores de enfermagem que atuam em regime de turnos, principalmente noturno, apresentam maiores prevalência de sintomatologia somática e, entre elas, alteração da qualidade do sono, distúrbios digestivos e sintomas de fadiga ${ }^{23}$.

Acerca da queixa dores de cabeça frequentes, estudo realizado com trabalhadores de enfermagem de três hospitais gerais constatou que das 65 notificações de doenças do sistema nervoso diagnosticadas, 39 eram cefaleia e 21 enxaqueca. Além de a cefaleia acarretar agravos para a saúde do indivíduo devem-se considerar os encargos financeiros, pois esta condição é responsável por $20 \%$ do absenteísmo em instituições de saúde no Brasil ${ }^{24}$.

Quanto ao fator IV (pensamentos depressivos), 31,8\% residentes afirmaram ter perdido o interesse pelas coisas, $17,6 \%$ indicaram ser incapaz de desempenhar um papel útil em sua vida, 6,9\% apontaram se sentir inútil em sua vida e 3,1\% mencionaram ter pensado dar fim a sua vida, (Tabela 2). Esses resultados são bastante preocupantes, principalmente ao se analisar o perfil da amostra, composto majoritariamente por jovens, do sexo feminino, solteiros e cuja característica da profissão é o convívio com a dor, a morte e o sofrimento das pessoas.

Estudo sobre a depressão em trabalhadores de enfermagem evidenciou a associação com algumas características sociodemográficas (ser solteiro, jovem adulto, maior escolaridade e baixa renda familiar); aspectos relacionados à organização do trabalho (trabalho noturno, sobrecarga, conflitos no relacionamento interpessoal, baixa autonomia e insegurança no desenvolvimento das atividades) e conflitos familiares. A depressão por si só é considerada um risco para o suicídio, sendo a probabilidade maior naqueles indivíduos que já apresentam reações depressivas e com nível elevado de cansaço emocional, alta despersonalização e baixa realização profissional. Na gênese da depressão, devemse também considerar alguns fatores externos ao trabalhador como a sobrecarga de trabalho, os baixos salários, a carga horária excessiva, o desgaste e a preocupação com o desenvolvimento do trabalho ${ }^{25}$.

Portanto, os trabalhadores devem reconhecer os fatores de risco para o adoecimento psíquico, de modo a intervir na perspectiva da promoção da saúde, acrescentados aos investimentos das instituições em dispositivos de educação permanente e em avaliações ou exames periódicos de saúde, manutenção de ambientes de trabalho saudáveis e suporte para ações de reorientação de comportamentos e hábitos que favoreçam o bem-estar e a qualidade de vida ${ }^{19}$.

\section{CONCLUSÃO}

O alto percentual de suspeição de TMC em residentes de enfermagem, cuja amostra foi composta majoritariamente por jovens, solteiros e do sexo feminino, encontra-se bem acima da prevalência identificada em outros estudos com profissionais da área da saúde, devendo-se considerar outras variáveis como as poucas horas de sono, o uso de medicamentos e o afastamento do ambiente de formação para tratamento de problemas de saúde.

A maioria dos participantes afirmou se sentir nervosas, tensas e preocupadas, cansadas o tempo todo e dormir mal. Tais resultados devem levar em conta algumas limitações metodológicas - a condição da pesquisa transversal, sem definição ou estabelecimento do nexo causal; e a amostra reduzida restrita a uma única instituição, e que impede a generalização dos achados. Tais limitações podem fomentar novos estudos dessa natureza, em amostra representativa do universo, de modo a estimar a prevalência de suspeição de TMC e a associação com as variáveis sociodemográficas, ocupacionais e a sua influência na saúde mental.

Os resultados obtidos servem de alerta para docentes e preceptores de instituições de ensino que com programas de residência, devido ao risco de desenvolvimento de transtornos psíquicos severos, com repercussões negativas para a formação, o processo de cuidar e a qualidade de vida do residente. Há necessidade de ações de cunho preventivo e terapêutico no sentido de minimizar o sofrimento psíquico, tendo em vista que problemas de saúde referidos pelos residentes podem interferir negativamente na prestação de cuidados seguros e no relacionamento interpessoal.

\section{REFERÊNCIAS}

1. Who MP. A user's guide to the self reporting questionnaire (SRQ). Geneva (SUI): Division of Mental Health Organization. 1994. [cited 2018 Jan 7]. Available from: http://apps.who.int/iris/bitstream/10665/61113/1/WHO_MNH_PSF_94.8.pdf

2. Machado MH, Oliveira E, Lemos W, Lacerda WF, Filho Wilson A, Wermelinger $M$ et al. Mercado de trabalho da enfermagem: aspectos gerais. Enferm. Foco [internet] 2016 [cited 2018 Mar] 7(esp):35-62. Available from: http://biblioteca.cofen.gov.br/wpcontent/uploads/2016/07/Mercado-de-trabalho-da-enfermagem-aspectos-gerais.pdf

3. Baptista ATP, Souza NVDO, Gallasch CH, Varella TCMML, Noronha IR, Noronha IR. Illness among nursing workers in the hospital contexto. Rev. enferm. UERJ. 2018 [cited 2019 Aug 14]; 26:e31170. DOI: https://doi.org/10.12957/reuerj.2018.31170 
4. Rocha SH, Bussinger ECA. The invisibility of occupational mental diseases in the contemporary labor world. Rev. pensar. 2016 (cited 2018 Aug 12]; 21(3):1104-22 DOI: http://dx.doi.org/10.5020/2317-2150.2016.v21n3p1104

5. Oliveira EB, Carvalho RAC, Teixeira E, Zeitoune RCG, Saboia VM, Gallasch CH. Factors involved in the training of resident nurses: view of alumni from a residency program. Rev. min. enferm. 2017 [cited 2018 Nov 1];21(e-1064): 1-7. DOI: http://dx.doi.org/10.5935/1415-2762.20170074

6. Carvalho DB, Araujo TM, Bernardes KO. Common mental disorders in primary health care workers. Rev. bras. saude. ocup. 2016 [cited 2018 May 08]; 41e27. DOI: http://dx.doi.org/10.1590/2317-6369000115915

7. Fernandes MA, Soares LMD, Silva JS. Work-related mental disorders among nursing professionals: a brazilian integrative review. rev. brasil. de med. do trab. 2018 [cited 2018 Feb 14]; 27(3):38-5. DOI: https://doi.org/10.5327//1679443520180228.

8. Martins JT, Ribeiro RP, Remijo KP, Ribeiro PHV. Mental disorders linked to the nursing work. Rev. enferm. UFPE on line. 2014 [cited 2018 Feb 21]; 8(6):1746-56. Available from: DOI: https://periodicos.ufpe.br/revistas/revistaenfermagem/article/view/13650

9. Conselho Federal de Enfermagem. Resolução COFEN no 259/2001 [site da Internet]. Estabelece padrões mínimos para registro de enfermeiro especialista, na modalidade de residência em enfermagem. Brasília (DF): COFEN, 2001. [cited 2018 Feb 20] Available from: http://www.portalcofen.com.br/legislacao/resoluções

10. Mari JJ, Willians P. A validity study of a psychiatric screening questionnaire (SRQ-20) in primary care in the city of São Paulo. Jornal bras. psiq. 1986 [cited 2018 Mar 07]; 148:23-6. Available from: https://www.ncbi.nlm.nih.gov/pubmed/3955316

11. Gonçalves DM, Stein AT, Kapczinski F. Performance of the Self-Reporting Questionnaire as a psychiatric screening questionnaire: a comparativestudy with Structured Clinical Interview for DSM-IV-TR. Cad. Saúde Pública. 2008 [cited 2018 Jan 21]; 24(2):380-90. DOI: http://dx.doi.org/10.1590/S0102-311X2008000200017

12. Lima LSV, Oliveira EB, Mauro MYC, Lisboa MTL, Assad LG, Carvalho RAC. Psychosocial risks in specialized units: implications for the training and health of nurse residents. Rev. enferm. UERJ. 2015 [cited 2018 Sep 17]; 23(2): 229-34. DOI: http://doi.org/10.12957/reuerj.2015.16507

13. Perez Junior EF, Oliveira EB. Technological innovations in intensive care unit: effects on nursing staff health. Rev. enferm. atual. 2016 [cited 2018 Oct 17]; 77:9-13. Available from: https://www.revistaenfermagematual.com.br/index.php/revista/article/view/368/250

14. Carvalho CN, Melo-Filho DA, Carvalho JAG, Amorim ACG. Prevalence and factors associated with common mental disorders in medical multiprofissional health residents. Jornal bras. psiq. 2013 [cited 2018 Jan 20]; 62(1):38-5. DOI: http://dx.doi.org/10.1590/S0047-20852013000100006.

15. Ansolin AGA, Rocha DLB, Santos RP, Pozzo VCD. Prevalence of common mental disorder between psycholgy and nursing students. Arq. Ciênc. Saúde. 2015 [cited 2018 Oct 21]; 22(1) 42-5. Available from: http://www.cienciasdasaude.famerp.br/index.php/racs/article/view/83/103

16. Silva KL, Sena RR, Tavares TS, Belga SMMF, Maas LW. Migrant nurses in Brazil: demographic characteristics, migration flow and relationship with the training process. Rev. latinoam. enferm. (Online). 2016 [cited 2018 Oct 15]; 24:e2686. DOI: http://dx.doi.org/10.1590/1518-8345.0390.2686

17. Urbaneto JS, Magalhães MCC, Maciel VO, Sant'Anna VM, Gustavo AS, Figueiredo CEP, etal Work-related stress according to the demand-control model and minor psychic disorders in nursing workers. Esc. Enferm. USP. 2013 [cited 2018 Feb 08]; 47(3):118693. DOI: https://dx.doi.org/10.1590/S0080-623420130000500024

18. Fernandes DM, Marcolan JF. Work and depression symptoms in family health strategy nurses. SMAD. Revista eletrônica saúde mental álcool e drogas. 2017 [cited 2018 Jul 24];13(1):37-4. DOI: https://dx.doi.org/10.11606/issn.1806-6976.v13i1p37-44

19. Magnago TSBS, Prochnow A, Urbaneto JS, Greccco PBT, Beltrame M, Mancio E, Luz. Relationship between work ability in nursing and minor psychological disorders. Texto \& contex enferm. 2015 [cited 2018 Mar 12]; 24(2): 362-70. DOI: http://dx.doi.org/10.1590/0104-07072015002580013

20. Sousa KHJF, Lopes DP, Tracera GMP, Abreu AMM, Portela LF, Zeitoune RCG. Common mental disorders among nursing workers in a psychiatric hospital. Acta Paul. Enferm. (Online). 2019[cited 2019 Aug 14]; 32(1):1-10. DOI: http://dx.doi.org/10.1590/19820194201900002

21. Oliveira EB, Souza NVM, Chagas SCS, Lima LSV, Correa RA. Effort and reward in the work of nurse resident in specialized units. Rev. enferm. UERJ. 2013 [cited 2018 Oct 09]; 21(2): 173-8. Available from: http://www.facenf.uerj.br/v21n2/v21n2a06.pdf

22. Alvim CCE, Souza MMT, Gama LN, Passos JP. Relationship between the work process and mental illness nursing staff. Revista Fluminense de Extensão Universitária. 2017 [cited 2018 Sep 12]; 07 (1): 12-16. Available from: http://editora.universidadedevassouras.edu.br/index.php/RFEU/article/view/918/717

23. Souza APC, Passos JP. The disturbance of sleep disorders in nursing professionals. Revista lberoamericana de Saúde e Envelhecimento. 2015 [cited 2018 Nov 30]; 1(2): 178-90. Available from: http://www.revistas.uevora.pt/index.php/saude_envelhecimento/article/view/61/86

24. Guimarães ALO, Felli VEA. Notification of health problems among nursing workers in university hospitals. Rev. bras. enferm. (Online). 2016 [cited 2018 Dec 21]; 69(3):507-14. DOI: http://dx.doi.org/10.1590/0034-7167.2016690313i

25. Silva DSD, Tavares NVS, Alexandre ARG, Freitas DA, Brêda MZ, Albuquerque MCS et al. Depression and risk of suicide in professional nursing: integrative review. Esc. Enferm. USP. 2015 [cited 2018 Apr 6]; 49(6):1027-36. DOI: http://dx.doi.org/10.1590/S0080-623420150000600020 